The role of chromatin structure in gene regulation of the human malaria parasite

Gayani Batugedara ${ }^{1}$, Xueqing M. Lu ${ }^{1}$, Evelien M. Bunnik ${ }^{2}$, and Karine G. Le Roch ${ }^{1 *}$

${ }^{1}$ Department of Cell Biology and Neuroscience, University of California Riverside, Riverside, CA 92521, USA

${ }^{2}$ Department of Microbiology, Immunology \& Molecular Genetics, The University of Texas Health Science Center at San Antonio, San Antonio, TX 78229, USA

* Corresponding author:

Karine G. Le Roch

Institute for Integrative Genome Biology

Center for Disease Vector Research

Department of Cell Biology and Neuroscience

University of California Riverside

900 University Avenue

Riverside, CA 92521

USA

Tel: +1 (951) 827-5422

Fax: +1 (951) 827-5155

E-mail: karine.leroch@ucr.edu

\title{
Keywords:
}

Epigenetics; gene regulation; chromatin; nucleosome; Plasmodium; malaria 


\begin{abstract}
The human malaria parasite, Plasmodium falciparum, depends on a coordinated regulation of gene expression for development and propagation within the human host. Recent developments suggest that gene regulation in the parasite is largely controlled by epigenetic mechanisms. Here, we discuss recent advancements contributing to our understanding of the mechanisms controlling gene regulation in the parasite, including nucleosome landscape, histone modifications and nuclear architecture. In addition, various processes involved in regulation of parasite-specific genes and gene families are examined. Finally, we address the use of epigenetic processes as targets for novel antimalarial therapies. Collectively, these topics highlight the unique biology of $P$. falciparum, and contribute to our understanding of mechanisms regulating gene expression in this deadly parasite.
\end{abstract}




\section{The malaria parasite}

2 The human malaria parasite remains one of the deadliest infectious agents worldwide. In

3 2015, an estimated 214 million cases of infection and 438,000 malaria-related deaths

4 were reported [1]. Most malaria infections occur in sub-Saharan Africa; however

5 developing countries in South East Asia and South America are also affected. Children

6 under the age of five and pregnant women are most susceptible to the disease and in

72015 , children under the age of five accounted for approximately $70 \%$ of all malaria-

8 related deaths.

10 Plasmodium falciparum, one of five Plasmodium species that can infect humans, is

11 responsible for the most severe disease symptoms and the highest mortality rate in

12 humans. The parasite develops through a complex life cycle that involves two hosts; the

13 Anopheles mosquito and the human host (Figure 1). The parasite's life cycle begins as an

14 infected Anopheles mosquito takes a blood meal from a human and in the process injects

15 sporozoites into the host bloodstream. The sporozoites translocate to the liver, invade

16 liver cells (hepatocytes) and replicate multiple times for a two-week period, producing

17 thousands of merozoites that leave the liver and invade red blood cells (erythrocytes) [2,

$183]$.

20 During the intra-erythrocytic developmental cycle (IDC), the parasite develops asexually

21 through ring, trophozoite and schizont stages and multiplies by a process of replication

22 termed schizogony. As the parasite progresses through the three distinct developmental

23 stages, it undergoes multiple rounds of nuclear replication and cytokinesis to produce 16- 
2432 daughter cells at the end of each IDC. The daughter merozoites then burst out of the

25 host red blood cell and invade new healthy red blood cells. During the IDC,

26 environmental stress can trigger the parasites into committing to sexual development

27 resulting in differentiation into male and female gametocytes. The mature gametocytes

28 can be ingested by a feeding mosquito, undergo sexual replication in the mosquito

29 midgut, and develop further into salivary gland sporozoites to be transmitted to a new

30 human host as the mosquito takes the next blood meal. This multi-stage life cycle of the

31 parasite is tightly regulated, most likely by strict control of stage-specific gene

32 expression. In eukaryotes, stage-specific regulation of gene expression can be a combined

33 effect of transcriptional, post-transcriptional and translational control. In P. falciparum,

34 the nature and the contribution of mechanisms regulating gene expression at the

35 transcriptional level, including the role of chromatin structure, are starting to emerge. In

36 this review, we summarize the current knowledge on the role of chromatin structure and

37 epigenetics in gene regulation of the human malaria parasite and its potential to identify

38 much-needed new therapies.

\section{$40 \quad P$. falciparum genome}

41 The human malaria parasite $P$. falciparum has a relatively compact genome of twenty

42 three million base pairs that is organized into fourteen chromosomes (per haploid

43 genome) [4]. The P. falciparum genome is the most AT-rich eukaryotic genome

44 sequenced to date, with an overall AT-composition of $\sim 80 \%$, rising to $90-95 \%$ in introns

45 and intergenic regions. The distinct developmental stages of the $P$. falciparum life cycle

46 (Figure 1) are characterized by coordinated changes in gene expression [5-10]. In 
47 eukaryotes, gene expression is partly controlled by transcription factors that bind to cell-

48 or tissue-specific promoters to regulate transcription [11]. However, a surprisingly low

49 number of specific transcription factors have been identified in the parasite genome [12,

50 13] and in particular, only a few stage-specific transcription factors have been validated

51 [14-20]. Therefore, the coordinated cascade of transcripts observed throughout the

52 parasite life cycle is unlikely to be regulated only by this limited collection of specific

53 transcription factors, and suggests that additional components and mechanisms, such as

54 post-transcriptional [21-25], translational and post-translational regulation [21, 26, 27] as

55 well as change of chromatin structure, may control the expression of the predicted 6,372

56 genes in the malaria parasite.

58 How DNA is packaged inside the nucleus greatly influences gene expression. After a

59 general overview of what is know in higher eukaryotes, we describe important features of

60 the $P$. falciparum nuclear and chromatin landscape and how these features, including

61 histone modifications, nucleosome occupancy and the three-dimensional (3D) nuclear

62 organization, may affect gene expression.

63

\section{Eukaryotic chromatin structure}

65 In a eukaryotic cell, genomic DNA is tightly wrapped around histone proteins and 66 assembled as nucleosomes. These nucleosomes are then coiled and packaged together

67 resulting in a fiber also known as chromatin. Interactions between chromatin and protein

68 complexes as well as the dynamics of nucleosome positioning and post-translational 
69 modifications (PTMs) of histone core proteins are of vital importance to the usage of

70 DNA.

72 The major step in gene transcription initiation is the recruitment of RNA polymerase II,

73 along with other general transcription factors (TFIIs), to promoter regions to form the

74 basal pre-initiation complex (PIC). Recent genome-wide nucleosome mapping studies in

75 model organisms, such as yeast and human, have revealed consensus patterns in

76 nucleosome organization, including lower nucleosome density at intergenic regions as

77 compared to genic regions, a strong nucleosome depleted region (NDR) near the

78 promoter, and well-positioned nucleosomes (i.e. -1 and +1 nucleosomes) containing

79 variant histone H2A.Z around the transcription start site (TSS) [28-31]. These findings

80 suggest that specific positioning of nucleosomes, especially at promoter and transcription

81 start or stop regions, largely contributes to transcriptional control by governing the access

82 of components of the transcription initiation machinery to their binding sites.

83 Furthermore, to ensure nucleosome dynamics and gene expression regulation,

84 nucleosome components or the entire nucleosome may be repositioned, removed or

85 replaced through the action of ATP-dependent chromatin remodeling enzymes. In

86 addition, post-translational modifications of histone proteins can have large effects on

87 chromatin structure and gene activity. For instance, acetylation of histone H3 at lysine 9

88 or 14 of their N-terminal tail (H3K9ac and H3K14ac) often alters the physical and

89 chemical stability of nucleosomes, resulting in an open chromatin structure and a

90 transcriptionally permissive state [32]. On the other hand, trimethylation of histone $\mathrm{H} 3$ at 
91 lysine 9 and 27 (H3K9me3 and H3K27me3) is often associated with a heterochromatin

92 state and repression of gene expression [33].

94 Besides precise positioning of nucleosomes, gene expression requires physical interaction

95 between promoter regions and their distal regulatory elements, yet promoters and their

96 regulatory elements are often linearly separated along the chromosome. To overcome this

97 spatial constraint, chromatin loops are formed to bring together the regulatory elements

98 and their promoters for gene activation. For example, the distal enhancer known as locus

99 control region (LCR) of beta-globin ( $\beta$-globin) genes makes contact with globin gene

100 promoters through chromatin looping and dynamically changes its interactions with the

101 promoters of embryonic, fetal, and adult $\beta$-globin genes to ensure expression of the

102 correct set of $\beta$-globin genes at the proper developmental stage [34-38]. Chromatin loops

103 can also play a role in gene silencing. For example, the maternal copy of the insulin-like

104 growth factor $2(\operatorname{Igf} 2)$ gene is silenced by placement in an inactive chromatin loop that

105 prevents enhancer-promoter interaction and allows the exclusive expression of the

106 paternal allele $[39,40]$.

108 Over the past decade, a series of molecular and genomic approaches have been developed 109 (3C, 4C, 5C, Hi-C, etc.) to study the higher order organization of chromosomes by 110 mapping interactions between genomic loci (see Box 1) [41]. Hi-C analyses of mouse and

111 human chromosome structures have revealed that eukaryotic genomes are organized into

112 large blocks that show high levels of chromatin interactions within that region, but not

113 with other loci in the genome. These regions, called topologically associated domains 
114 (TADs) [42, 43], are well defined by insulator proteins [44, 45] and are composed of

115 many chromatin loops that have important functional roles in regulating gene expression.

116 On a higher dimension, individual chromosomes organize and occupy distinct territories

117 within the nucleus, and such organization is highly associated with gene density; gene-

118 dense chromatin is usually enriched in the internal part of the nucleus, while gene-poor

119 regions tend to locate toward the nuclear periphery [46-53].

120

121 Emerging evidence shows that long non-coding RNAs (lncRNAs) are also involved in

122 the control of transcription and genome activity by affecting chromatin-remodeling

123 events, including nucleosome positioning and chromatin looping [54]. A well-known

124 example is the IncRNA known as Xist, which mediates X-chromosome inactivation

125 during zygotic development [55]. Deposition of Xist on the X chromosome recruits

126 histone-modifying enzymes that place repressive histone marks, such as H3K9 and

127 H3K27 methylation, leading to gene silencing and the formation of heterochromatin.

128 How many of these typical chromatin features are maintained in Plasmodium, as well as

129 the areas where additional research is necessary, will be highlighted in the upcoming 130 topics.

131

132 Chromatin structure and gene regulation in $P$. falciparum

133 Transcriptional machinery in Plasmodium

134 Since the publication of the P. falciparum genome in 2002 [4], researchers have 135 attempted to explore the transcriptional machinery of the parasite in detail. The basal

136 transcriptional machinery, RNA polymerase II and all its subunits have been identified in 
137 the parasite [13, 56]. Additionally, a total of 23 TFII components have been found.

138 Although four TATA-binding protein (TBP) associated factors (TAFs) have been

139 discovered in P. falciparum, the parasite seems to lack the classical TFIID subunits with

140 a histone fold domain. The histone fold domain allows the TAFs to assemble into

141 heterodimers [56]. In yeast, the TFIID complex contains the TATA-binding protein

142 (TBP) and TBP-associated proteins (TAFs) and more than half of these proteins contain a

143 histone fold motif [57]. The fact that the TAFs in P. falciparum do not contain this motif

144 suggests that the parasite TFIID complex is divergent from other eukaryotes. As so many

145 TAFs are missing in P. falciparum compared to other eukaryotes, alternative mechanisms

146 may be more important for transcriptional regulation in the parasite.

148 In eukaryotes, specific transcription factors (TFs) recruit and activate the transcription

149 pre-initiation complex. Remarkably, in P. falciparum, only about 30 specific transcription

150 factors have been identified [12, 13]. Twenty-seven of these TFs belong to the

151 apicomplexan-specific family of transcription factors and contain a modified form of the

152 AP2 domain found in plant TFs (ApiAP2) [12]. These TFs are present throughout the

153 parasite life cycle and are believed to control the transition between specific

154 developmental stages. Some examples include AP2-G for the development of

155 gametocytes [17, 18], AP2-Sp for sporozoite development [19], AP2-L for the

156 development of liver-stage parasites [16], and AP2-O for the development of ookinetes in

157 the mosquito [20]. Another member of the ApiAP2 family, PfSIP2, is shown to bind to

158 heterochromatic regions of the genome and act as a transcriptional repressor [58]. Despite

159 continued experimentation, it remains to be determined how ApiAP2 transcription factors 
160 recruit RNA polymerase II to sites of transcription. Similar to the lack of TAFs

161 mentioned above, the small number of specific TFs identified in P. falciparum highlights

162 the role of alternative mechanisms regulating gene expression.

163

164 It is now evident that most of the P. falciparum genome is maintained in a decondensed

165 chromatin environment called euchromatin, while only a small subset, including 166 subtelomeric regions and a few internal loci, are contained within highly condensed

167 heterochromatin cluster(s) [59, 60]. The heterochromatin cluster(s) of the parasite

168 genome are marked by H3K9me3 modifications and heterochromatin protein 1 (PfHP1),

169 and harbor gene families encoding clonally variant antigens (var, rifin, stevor and pfmc-

$1702 \mathrm{tm}$ ), invasion gene families ( $e b a$ and $\mathrm{clag}$ ) and a few other loci such as the gametocyte-

171 specific transcription factor pfap2-g during the IDC [61-65]. The presence of these

172 repressive marks on parasite stage-specific gene families suggests that mechanisms

173 regulating transcription of these genes may be more conserved with higher eukaryotes,

174 than the rest of the genes in the Plasmodium genome.

175

176 Epigenetic regulation of clonally variant gene families

177 P. falciparum virulence genes

178 Disease pathogenesis in malaria is the result of the parasite's ability to escape host

179 immune responses. The var gene family, the best characterized multi-gene family in $P$.

180 falciparum, encodes erythrocyte membrane proteins 1 (PfEMP1s) that are expressed at

181 the surface of the infected erythrocyte and play a key role in cytoadherence and antigenic

182 variation [66]. Approximately sixty var genes are present in a haploid genome of $P$. 
183 falciparum, but only one var gene is expressed at any given time [67]. By switching var

184 gene expression, the parasite is able to avoid host immune responses. The mechanisms

185 regulating var gene expression in P. falciparum in vitro have emerged recently and are

186 discussed below.

187

188 Histone post-translational modifications affecting virulence gene expression

189 Silent var genes are clustered to one or more repressive regions at the nuclear periphery, 190 marked by H3K9me3 and PfHP1 (Figure 2A) [59-63, 65, 68, 69]. Histone deacetylases

191 (HDACs), in particular NAD ${ }^{+}$-dependent class III HDAC proteins PfSIR2A and PfSIR2B

192 and class II HDAC protein PfHDA2, play a role in regulating the repressive clusters

193 containing silent var genes, as manipulated parasite lines lacking these proteins show loss

194 of monoallelic var gene expression [65, 70-72]. PfSET2 is a histone lysine

195 methyltransferase (HKMT) that specifically marks var genes, and the disruption of

196 PfSET2 results in the de-repression of the silenced var gene cluster(s) [73, 74]. Absence

197 of PfHP1 in the parasite has also been shown to result in loss of monoallelic var gene

198 expression as well as result in parasite growth arrest [75], which indicates that PfHP1

199 plays an essential role in maintaining repressive heterochromatin.

200

201 The active var gene, transcribed at the ring stage, is distinguished by the presence of

$202 \mathrm{H} 3 \mathrm{~K} 4 \mathrm{me} 3$ and $\mathrm{H} 3 \mathrm{~K} 9 \mathrm{ac}$ marks and resides in a region of the nucleus away from the 203 repressive heterochromatin cluster(s) $[65,70,76]$. At the later trophozoite and schizont

204 stages, the active var gene is controlled by the HKMT PfSET10, which is suggested to 205 play a role in maintaining epigenetic memory of var gene expression [77]. Collectively, 
206 these results highlight the relationship between proper chromatin assembly and regulation

207 of antigenic variation in the parasite.

209 Long non-coding RNAs

210 Another mechanism regulating the monoallelic expression of the var gene family is the

211 transcription of long non-coding RNAs (lncRNA). Two lncRNAs transcribed from a

212 bidirectional promoter within the var intron have been identified [78, 79]. Both these

213 IncRNAs are incorporated into chromatin after being capped but not polyadenylated.

214 Transcription of the sense lncRNA may play a role in positing the repressive histone

215 mark H3K36me3 at the var gene loci thereby functioning as a silencer of var gene

216 expression, while the antisense lncRNA is proposed to be required for expression of the

217 single active var gene [79].

218

219 Recently, a novel family of twenty-two long non-coding RNAs (lncRNAs) transcribed

220 from the telomere-associated repetitive elements (TAREs) was identified [80-82]. The

221 exact role of these lncRNAs is yet to be determined. However, these lncRNA TARE loci

222 are enriched with ApiAP2 transcription factor PfSIP2 binding sites. PfSIP2 has been

223 implicated in heterochromatin formation around subtelomeric var gene regions [58].

224 Therefore, lncRNA-TAREs may, directly or indirectly, help regulate var gene expression.

225 The TARE-lncRNAs show functional similarities to the eukaryotic family of non-coding

226 RNAs involved in telomere and heterochromatin maintenance [83], which further

227 validates the role for IncRNA-TAREs regulating heterochromatin and repressive centers

228 in the parasite genome. 
230 While in vivo studies of var gene regulation are challenging, results from human

231 infections suggest that infection-induced stress responses in the host, such as fever and

232 changes in bloodstream metabolites, can modify expression of PfEMP1 via changes in

233 histone modifications [84], confirming the importance of this epigenetic mechanism for

234 var gene regulation. Another in vivo study using patient samples found that not only do

235 the parasites switch between different PfEMP1 variants, but they also vary the expression

236 level of the PfEMP1 variants, most likely in response to host immune responses [85].

237 With these studies it is becoming clearer that the selection pressures in vitro are different

238 from those in vivo and epigenetic regulation of the clonally variant gene families may

239 contribute to the differences observed in vivo and in vitro.

241 P. falciparum invasion genes

242 Invasion of a new erythrocyte by the malaria parasite involves binding of parasite ligands

243 to specific recognition surface receptors on the red blood cell [86]. Eba, rhophl/clag,

$244 a c b p$ and $P f R H$ are among some of the gene families involved in the invasion process, but

245 are not essential for parasite survival. The genes in these families are thought to be

246 partially regulated through epigenetic mechanisms and show differential expression

247 patters in different parasite lines, as they can be in either active or inactive states [87]

248 (Figure 2B). According to a more recent study exploring the parasite-specific

249 bromodomain protein PfBDP1 using in vitro culture, invasion genes are regulated in a

250 more "classical" manner by transcription factors interacting with specific promoters [88].

251 In schizonts, an enrichment of PfBDP1 was observed at the transcription start sites of 
252 invasion genes. PfBDP1 was shown to positively regulate transcription of invasion genes

253 by binding to acetylated histone H3. Additionally, conditional knockdown of PfBDP1

254 resulted in erythrocyte invasion defects and parasite growth inhibition, further confirming

255 the essentiality of this bromodomain protein for the coordinated expression of invasion 256 genes in P. falciparum.

258 Epigenetic regulation of gametocytogenesis

259 As the malaria parasites continue asexual replication, a small fraction of parasites will

260 commit to sexual differentiation and form gametocytes with every replication cycle. It is

261 believed that this commitment is made during the schizont stage, however what prompts

262 the asexual stage parasites to commit to sexual differentiation is not well understood. The

263 AP2 transcription factor, pfap2-g, located on chromosome 12 , is one of the master

264 regulators of gametocyte differentiation $[17,18]$. In asexual parasites, the locus

265 containing pfap2-g is localized to the nuclear periphery and silenced by $\mathrm{H} 3 \mathrm{~K} 9 \mathrm{me} 3$ and

266 PfHP1 (Figure 2C) [65, 69]. In vitro studies show that down-regulation of PfHDA2

267 activates pfap2-g and induces the formation of gametocytes [72]. Similarly, depletion of

268 PfHP1 activates pfap2-g and increases the rate of gametocyte production [75]. However,

269 these observations have not been confirmed in vivo.

270

271 Histones and nucleosome landscape of $\boldsymbol{P}$. falciparum

272 Recently, high-sensitivity mass spectrometry experiments have identified a total of 232

273 different histone post-translational modifications (PTMs) during the P. falciparum

274 intraerythrocytic stages, including acetylations, methylations, phosphorylations, 
275 ubiquitylations and sumoylations [89]. Many of these histone PTMs had never been

276 detected in Plasmodium or in other organisms and their exact function remains to be

277 determined. It is however important to mention that a majority of the parasite genome

278 carries a large proportion of activating histone marks (H3K9ac and H3K4me3) compared

279 to silencing marks (H3K9me3 and H3K36me3). This contrasts with what has been

280 identified in multicellular eukaryotes [90], but validates further the transcriptionally

281 permissive euchromatic state of the parasite genome. In mammalian genomes, H3K9ac

282 and H3K4me3 strictly localizes to active promoters [91-95], while in P. falciparum these

283 modifications not only mark promoters and 5' coding regions of genes that are highly

284 transcribed [96, 97], but are also found in intergenic regions and 'silenced' promoters

$285[61,96,98]$.

286

287 The nucleosome landscape of $P$. falciparum is similar to other eukaryotes in some

288 aspects. First, the parasite genome exhibits a depletion of nucleosome coverage in the

289 promoter regions of genes [99-101], most likely to allow binding of transcription factors

290 and other components of the transcription machinery. Second, lower levels of

291 nucleosomes are observed in intergenic regions compared to coding regions [100-102].

292 Some studies dispute this finding and attribute the difference in nucleosome levels to

293 sequencing biases introduced by the high AT-content of the intergenic regions [103]. In

294 these studies, the authors normalize the nucleosome coverage data using sonicated

295 genomic DNA, but because sonication tends to degrade AT-rich sequences faster than

296 GC-rich sequences, normalization using sonicated genomic DNA may over-correct for

297 AT-rich sequencing biases. Alternative methodologies that enrich for nucleosome 
298 depleted regions such as FAIRE-Seq [102] or ATAC-Seq [104] should be considered to

299 better evaluate sequencing biases of nucleosome positioning in an AT-rich genome.

300 Indeed, lower nucleosome levels are observed in intergenic regions of all other

301 eukaryotes [105-108] including Tetrahymena thermophila, another organism with an AT-

302 rich genome [109]. Third, genes with higher transcription levels exhibit a more open

303 chromatin structure at their core promoter region than silenced genes [99, 100, 103].

304 However, the nucleosome landscape of $P$. falciparum also displays some unique

305 characteristics (Figure 3). First, the parasite genome lacks a strongly positioned +1

306 nucleosome that marks the TSS [99, 100] and instead, the most strongly positioned

307 nucleosomes are found at the start and stop of the coding region. Second, the high AT-

308 content of the P. falciparum genome is likely to be the cause for the parasite-specific

309 nucleosome landscape as AT-rich DNA is relatively inflexible and thus does not easily

310 wrap around the nucleosome core. To solve this issue, the parasite has evolved its histone

311 variants H2A.Z and H2B.Z that bind weakly but more effectively to AT-rich DNA [110,

312 111]. H2A.Z and H2B.Z are found throughout intergenic regions of the parasite genome,

313 instead of being only present at the +1 nucleosome in other eukaryotes.

315 Another unique feature of the $P$. falciparum nucleosome landscape, that is also an area of 316 debate, is that nucleosome levels change as the parasite develops through the asexual 317 replication cycle. Other studies propose a more sequence-driven and transcription-

318 independent nucleosome positioning in the parasite [103]. These controversies seem to be 319 stemming from discrepancies in data normalization. When nucleosome occupancy 320 profiles are normalized by input parasite content or by the number of nuclei, these 
321 opposing datasets display similar nucleosome dynamics throughout the parasite life

322 cycle. Additionally, changes in nucleosome occupancy during parasite development have

323 also been confirmed by alternative approaches. Experiments including western blots

324 [112], mass spectrometry [89, 100, 113], MNase-Seq, FAIRE-Seq [102], and ChIP-seq

325 [100] showed that histone levels are lower during the transcriptionally active trophozoite

326 stage. This observation has prompted a model for gene regulation in the parasite genome

327 where nucleosome eviction drives the massive transcriptional event observed at the

328 trophozoite stage, which is followed by the schizont stage where the genome repacks in

329 preparation for re-invasion. At the schizont stage, nucleosomes are re-assembled and

330 global histone levels are restored to the levels observed before the transcriptionally active

331 trophozoite stage. At this later stage, regulation of transcription at the initiation level may

332 be controlled by more classical mechanisms of regulation such as stage-specific

333 transcription factors (AP2) and histone PTMs [88].

335 P. falciparum nuclear architecture

336 Much like in complex metazoans, the three-dimensional (3D) genome structure of $P$.

337 falciparum plays important roles in regulating gene expression. Initial observations of

338 global chromatin arrangement within the parasite nucleus were studied using

339 immunofluorescence microscopy and fluorescence in situ hybridization (FISH)

340 experiments $[65,114,115]$. Earlier FISH experiments revealed that var genes localize to

341 two to five clusters around the parasite nucleus [60, 65]. More recent studies showed a

342 single locus for the var gene-associated repressive cluster marked by H3K9me3 and

343 PfHP1 [116], as well as H3K36me3, which is mark of both active and silent var genes 
344 [74]. These observations were confirmed with the recent advancement of chromosome

345 conformation capture techniques such as Hi-C that capture genome-wide intra- and

346 interchromosomal interactions. However, because Hi-C captures the nuclear architecture

347 at the population level, unlike FISH that is performed at the single-cell level, Hi-C data

348 cannot be used to distinguish between multiple var gene clusters if the genes are

349 randomly distributed from cell to cell. In addition, technical challenges such as cell cycle

350 timing as well as cross-linking strategies may contribute to the discrepancies between

351 these methodologies. Since 3C-based and imaging methods inherently measure different

352 aspects of the 3D genome, there will be instances where the two datasets appear to be

353 inconsistent [117]. Nevertheless, Hi-C and FISH experiments together with chromatin

354 immunoprecipitation followed by microarray or next generation sequencing technologies

355 (ChIP-on-Chip or ChIP-Seq), have contributed to the discovery of atypical chromatin

356 features in the parasite genome [59].

357

358 P. falciparum chromosomes are arranged into folded structures, which are attached at the

359 centromere with the two chromosomal arms folding over each other in a parallel

360 orientation [59]. The clustering of the centromeres and telomeres on opposite regions of

361 the nucleus is comparable to the 3D genome structure observed in the similarly sized

362 budding and fission yeast $[118,119]$. Unlike the yeast genome, $P$. falciparum has several

363 additional complex structures mostly generated by var genes located internally on five

364 out of fourteen chromosomes [59]. These internal var genes colocalize with the

365 subtelomeric var genes at the nuclear periphery by creating additional loops in the

366 chromosomes. 
368 Hi-C experiments [59] as well as advanced microscopy examination [120] throughout the

369 IDC reveal that the nuclear organization undergoes several distinct changes during its

370 developmental progression, most likely in order to accommodate the high level of

371 transcriptional activity necessary during these stages of the parasite life cycle. Together

372 with the expansion of the nucleus, which reaches the maximum size and volume at the

373 trophozoite stage, the number of nuclear pores increases from 3-7 pores at the ring stage

374 to $12-58$ pores at the trophozoite stage [120]. Nuclear pores are located next to

375 euchromatic areas, and the increased number of pores suggests a transcriptionally active

376 trophozoite stage with the need to facilitate messenger RNA transport into the cytoplasm.

377 As the parasite undergoes schizogony, the contents of the nucleus together with the pores

378 are distributed among the resulting daughter nuclei. Increased nuclear pores at the

379 trophozoite stage correlate with partial loss of chromosomal territories for more

380 chromosome intermingling [59]. Additional observations from Hi-C experiments include

381 similar expression profiles for genes that are located close to each other with

382 colocalization of genes that are silenced during the IDC but expressed at other stages

383 [59]. Taken together, these observations highlight the importance of understanding

384 mechanisms regulating the dynamic nuclear organization and their role in controlling 385 gene expression.

387 Using chromatin structure and epigenetic regulation as drug targets

388 As mentioned previously, the maintenance and regulation of the repressive

389 heterochromatin environment within the parasite nucleus is essential for parasite survival. 
390 In particular, conditional deletions of PfHP1 or PfHDA2 have been shown to cause

391 developmental arrest of blood stage parasites [72, 75]. Profound transcriptional changes

392 have been observed for parasites treated with the drug apicidin, a potent inhibitor of

393 HDACs [121]. Apicidin does so by causing hypermethylation of H3K9 and H4K8

394 residues, which leads to deregulation of the global transcriptional cascade. Disruption of

395 histone acetylation and methylation levels, and in particular HKMTs, have also shown to

396 interfere with parasite growth and survival, although so far only a few small molecules

397 potent enough to inhibit HKMT activity in P. falciparum have been identified $[122,123]$.

398 Targeting HDAC and HKMT classes of enzymes as antimalarial therapies is a promising

399 strategy. However, many eukaryotic histone-modifying enzymes share conserved

400 catalytic domains and when targeted for antimalarial therapies could be toxic to the

401 human host as well. Therefore, potential drug compounds should be studied extensively

402 before they can be considered as novel antimalarial therapies.

403

404 Concluding Remarks and Future Perspectives

405 P. falciparum uses a combination of different epigenetic mechanisms to regulate its gene

406 expression. However, our understanding of the parasite epigenome is far from complete

407 (see Outstanding Questions). Although most chromatin modifications used by the

408 parasite are also common to other eukaryotes, several features of chromatin regulation

409 are unique to P. falciparum. Exploring the underlying regulatory mechanisms of how the

410 repressive cluster(s) are established and maintained could lead to identification of

411 specific proteins important for chromatin regulation in the malaria parasite. At the

412 epigenetic level, as outlined above, the Plasmodium genome architecture points towards a 
413 binary structure, with the majority of the genome existing as transcriptionally permissive

414 euchromatin and a small subset of genes present in a transcriptionally silent

415 heterochromatin state. This heterochromatin cluster is localized at the periphery of the

416 nucleus and is characterized by high levels of H3K9me3 and H3K36me3 histone marks,

417 PfHP1 and high nucleosome density. The euchromatin environment harbors active genes,

418 including the single active var gene at the nuclear periphery, and is characterized by high

419 levels of H3K4me3 and H3K9ac histone modifications. During the asexual cycle, the

420 parasite nucleus and chromatin undergo drastic remodeling to accommodate the high

421 transcriptional activity at the trophozoite stage. Chromatin structure remains relatively

422 compact during the ring and schizont stages, but opens substantially during the

423 trophozoite stage. This open-and-close chromatin structure is also reflected at the

424 nucleosome landscape and global histone levels. As the nucleus expands in size, which

425 can also be visualized using immunofluorescence and Giemsa staining, the number of

426 nuclear pores increases greatly and distribute around the nucleus. As the parasite

427 transitions from the trophozoite stage to the schizont stage, the changes in nuclear

428 architecture are reversed by reassembling nucleosomes, increasing global histone levels

429 and compacting the genome. In the transition from trophozoite to schizont stage, the

430 DNA is replicated, and the nucleus is divided into multiple daughter nuclei, each with a

431 small number of the nuclear pores previously present in the nucleus. Collectively, these

432 observations suggest that the majority of the parasite genome is regulated via genome-

433 wide changes in chromatin structure, while a small subset of genes are regulated by

434 classical transcriptional regulation mechanisms, such as changes in local chromatin

435 structure and specific transcription factors. Exploration of regulatory mechanisms that 
436 regulate large chromatin rearrangements throughout the parasite life cycle could enable

437 the discovery of molecules that can target parasite development with high specificity.

438

439

440

441

442 ACKNOWLEDGEMENTS

443 This work was financially supported by the National Institutes of Health (grants R01

444 AI85077-01A1 and R01 AI06775-01 to KGLR), the National Science Foundation (grant

445 IIS-1302134 to KGLR), the University of California, Riverside (USDA-NIFA-Hatch-

446225935 to KGLR), and The University of Texas Health Science Center at San Antonio

447 (EMB). The funding bodies had no role in the design of the study, in collection, analysis,

448 and interpretation of data, or in writing the manuscript. 


\section{Box 1. Selected methods for assaying 3D chromatin structure}

451 Chromosome conformation capture (3C) method identifies pairwise interactions

452 between two selected fragments in a one-to-one approach. The major steps of the $3 \mathrm{C}$

453 protocol include cross-linking cells with formaldehyde, digesting chromatin with a

454 restriction enzyme, ligating chromatin fragments that are in close proximity, reversal of

455 cross-links, and identifying the interaction frequency of the targeted regions using

456 primers specific to those regions in a quantitative polymerase chain reaction (PCR). For

457 3C experiments, prior knowledge of which genomic loci are likely to interact is required,

458 and the technique is best suited to study small numbers of loci or relatively small

459 genomic regions [124].

461 Circularized chromosome conformation capture/ chromosome conformation

462 capture-on-chip (4C) method uses high throughput sequencing to identify all contacts

463 for a selected locus in a one-versus-all approach. The 4C methodology is similar to 3C up

464 to the reverse crosslinking step. Following an additional round of digestion, DNA

465 fragments are self-ligated to create circular DNA containing both the locus of interest and

466 its interacting partner. Using inverse PCR, the interacting partner sequence is amplified

467 and sequenced, generating a genome-wide interaction profile for the region of interest.

468 The $4 \mathrm{C}$ technique is suitable for studying both inter and intrachromosomal contacts of a

469 specific region at high resolution [125].

471 Chromosome conformation capture carbon copy (5C) method is a many-to-many 472 approach capturing chromosome interactions for multiple loci in one experiment. This 473 methodology uses a pool of primers that are hybridized to different restriction sites in the 474 genome. During the ligation step, primer pairs on interacting fragments can be ligated 475 together. Each primer contains a universal sequence at its $5^{\prime}$ end to allow the 476 amplification of these ligation products, thus detecting the interaction frequencies for 477 multiple loci or genomic regions. One major bottleneck for $5 \mathrm{C}$ is the requirement for the 478 design of individual primers for each restriction site [126]. 

method provides true genome-wide capture of chromosome interactions, also described as an all-to-all approach. Hi-C follows the basic steps of $3 \mathrm{C}$ procedures with a modified

483 ligation step: after restriction digestion of cross-linked DNA, biotin-labeled nucleotides

484 are incorporated into the overhangs followed by blunt end ligation. The ligated fragments 485 are then isolated using streptavidin-coated magnetic beads and sequenced on a next486 generation sequencing platform. Hi-C provides a complete genome-wide interaction map, 487 including both local and long-range intrachromosomal contacts as well as 488 interchromosomal contacts; however, large amounts of sequencing data and complex 489 computational analyses are required [114].

494 Figure 1. Schematic representation of the life cycle of the malaria parasite. In the

495 human host, the parasite first develops through the liver stage, followed by subsequent

496 48-hour replication cycles inside red blood cells, which is the stage responsible for 497 symptomatic disease. During this replication cycle, a small proportion of parasites will 498 commit to sexual differentiation into male and female gametocytes that can be taken up 499 by a mosquito. Sexual reproduction takes place inside the mosquito midgut and 500 ultimately results in the formation of sporozoites that can be transmitted to a new human 501 host.

503 Figure 2. Epigenetic regulation of specific genes and gene families in Plasmodium

504 falciparum. (A) The family of var genes is controlled by clustering of silent var genes at

505 the nuclear periphery and the deposition of repressive H3K9me3 marks, which recruits

506 PfHP1 and results in the formation of heterochromatin. The single active var gene is 
507 isolated from all other var genes, marked by H3K4me3 and H3K9ac, and localized in a

508 euchromatic environment. LncRNAs transcribed from a bidirectional promoter in the var

509 introns also contribute to regulation of var gene expression. (B) Several families of

510 invasion genes are epigenetically regulated through repressive and active histone marks

511 that recruit heterochromatin marker PfHP1 and gene activator PfBDP1, respectively. (C)

512 During the IDC, gametocyte-specific TF pfap2-g localizes to the nuclear periphery and is

513 silenced by repressive histone marks, including H3K9me3, and PfHP1. Abbreviations:

514 IDC, intra-erythrocytic developmental cycle; HDA2, histone deacetylase 2; H3K9me3,

515 trimethylation of histone $\mathrm{H} 3$ at lysine 9; $\mathrm{H} 3 \mathrm{~K} 9 \mathrm{ac}$, acetylation of histone $\mathrm{H} 3$ at lysine 9;

516 LncRNAs, long non-coding RNAs; PfBDP1, bromodomain protein; PfHP1,

517 heterochromatin protein 1; SET2, histone lysine methyltransferase that specifically marks

518 var genes; SIR2A, silent information regulator 2A; SIR2B, silent information regulator

519 2B; TF, transcription factor; pfap2-g, gametocyte-specific transcription factor.

520

521

522 Figure 3. Differences in chromatin and genome organization between

523 Saccharomyces cerevisiae and Plasmodium falciparum. (A) The genome of $P$.

524 falciparum is more AT-rich compared to the similarly sized budding yeast. (B) In yeast,

525 the promoter region is flanked by strongly positioned nucleosomes $(-1$ and +1$)$. These

526 nucleosomes often contain the histone variant H2A.Z, which destabilizes the nucleosome

527 and promotes gene activation. The nucleosome landscape at the end of the gene mirrors

528 the landscape at the gene start. The nucleosome landscape around $P$. falciparum genes

529 shows strongly positioned nucleosomes at the start and stop of the coding region, and 
530 histone variants H2A.Z and H2B.Z throughout the intergenic regions. This parasite-

531 specific nucleosome landscape is most likely the result of the high AT-content of the

532 parasite genome. As AT-rich DNA is relatively rigid and does not easily wrap around a

533 nucleosome core the parasite may have partially solved this issue by evolving its histone

534 variants H2A.Z and H2B.Z to become more efficient at binding AT-rich DNA. (C) In

535 yeast, the process of transcription involves the local displacement of nucleosomes to

536 allow the transcription machinery to progress along the gene. In the malaria parasite,

537 nucleosome levels decrease dramatically during the trophozoite stage to facilitate massive

538 transcription. This global nucleosome eviction may contribute to a general

539 transcriptionally permissive state of the genome, allowing the efficient transcription of

$54070-80 \%$ of all P. falciparum genes at this stage of its life cycle. (D) S. cerevisiae

541 chromosomes have a folded architecture anchored at the centromere, with clustering of

542 the centromeres and telomeres on opposite sites of the nucleus. In P. falciparum, clusters

543 of var genes that are located internally on chromosomes colocalize with subtelomeric var

544 genes, resulting in additional looping structures in five out of fourteen chromosomes.

545 This contributes to a more complex genome organization than what is observed in yeast.

546 Abbreviations: NDR, nucleosome depleted region; PIC, pre-initiation complex; TSS,

547 transcription start site.

548

549

550

551

552 


\section{References}

554 1. WHO (2015) World Malaria Report. 2015.

555 http://www.who.int/malaria/publications/world-malaria-report-

556 2015/report/en/.

557 2. Rosenberg, R., et al. (1990) An estimation of the number of malaria sporozoites

558 ejected by a feeding mosquito. Transactions of the Royal Society of Tropical Medicine

559 and Hygiene 84, 209-212

560 3. Yuda, M. and Ishino, T. (2004) Liver invasion by malarial parasites--how do malarial

561 parasites break through the host barrier? Cellular microbiology 6, 1119-1125

562 4. Gardner, M.J., et al. (2002) Genome sequence of the human malaria parasite

563 Plasmodium falciparum. Nature 419, 498-511

564 5. Le Roch, K.G., et al. (2003) Discovery of gene function by expression profiling of the 565 malaria parasite life cycle. Science 301, 1503-1508

566 6. Bozdech, Z., et al. (2003) The transcriptome of the intraerythrocytic developmental

567 cycle of Plasmodium falciparum. PLoS biology 1, E5

568 7. Bunnik, E.M., et al. (2013) Polysome profiling reveals translational control of gene

569 expression in the human malaria parasite Plasmodium falciparum. Genome biology 14,

$570 \quad \mathrm{R} 128$

571 8. Otto, T.D., et al. (2010) New insights into the blood-stage transcriptome of

572 Plasmodium falciparum using RNA-Seq. Molecular microbiology 76, 12-24

573 9. Lopez-Barragan, M.J., et al. (2011) Directional gene expression and antisense

574 transcripts in sexual and asexual stages of Plasmodium falciparum. BMC genomics 12, $575 \quad 587$

576 10. Rovira-Graells, N., et al. (2012) Transcriptional variation in the malaria parasite

577 Plasmodium falciparum. Genome research 22, 925-938

578 11. Consortium, E.P. (2012) An integrated encyclopedia of DNA elements in the human

579 genome. Nature 489, 57-74

580 12. Balaji, S., et al. (2005) Discovery of the principal specific transcription factors of

581 Apicomplexa and their implication for the evolution of the AP2-integrase DNA binding

582 domains. Nucleic acids research 33, 3994-4006

583 13. Coulson, R.M., et al. (2004) Comparative genomics of transcriptional control in the

584 human malaria parasite Plasmodium falciparum. Genome research 14, 1548-1554

585 14. Young, J.A., et al. (2008) In silico discovery of transcription regulatory elements in

586 Plasmodium falciparum. BMC genomics 9, 70 
587 15. Campbell, T.L., et al. (2010) Identification and genome-wide prediction of DNA

588 binding specificities for the ApiAP2 family of regulators from the malaria parasite. PLoS

589 pathogens 6 , e 1001165

590 16. Iwanaga, S., et al. (2012) Identification of an AP2-family protein that is critical for

591 malaria liver stage development. PloS one 7, e47557

592 17. Kafsack, B.F., et al. (2014) A transcriptional switch underlies commitment to sexual

593 development in malaria parasites. Nature 507, 248-252

594 18. Sinha, A., et al. (2014) A cascade of DNA-binding proteins for sexual commitment

595 and development in Plasmodium. Nature 507, 253-257

596 19. Yuda, M., et al. (2010) Transcription factor AP2-Sp and its target genes in malarial 597 sporozoites. Molecular microbiology 75, 854-863

598 20. Yuda, M., et al. (2009) Identification of a transcription factor in the mosquito-

599 invasive stage of malaria parasites. Molecular microbiology 71, 1402-1414

600 21. Kirchner, S., et al. (2016) Recent advances in malaria genomics and epigenomics.

601 Genome medicine 8, 92

602 22. Balu, B., et al. (2011) CCR4-associated factor 1 coordinates the expression of

603 Plasmodium falciparum egress and invasion proteins. Eukaryotic cell 10, 1257-1263

604 23. Bunnik, E.M., et al. (2016) The mRNA-bound proteome of the human malaria 605 parasite Plasmodium falciparum. Genome biology 17, 147

606 24. Eshar, S., et al. (2015) PfSR1 controls alternative splicing and steady-state RNA 607 levels in Plasmodium falciparum through preferential recognition of specific RNA 608 motifs. Molecular microbiology 96, 1283-1297

609 25. Vembar, S.S., et al. (2015) The PfAlba1 RNA-binding protein is an important 610 regulator of translational timing in Plasmodium falciparum blood stages. Genome biology 61116,212

612 26. Caro, F., et al. (2014) Genome-wide regulatory dynamics of translation in the 613 Plasmodium falciparum asexual blood stages. eLife 3:e04106

614 27. Foth, B.J., et al. (2008) Quantitative protein expression profiling reveals extensive 615 post-transcriptional regulation and post-translational modifications in schizont-stage 616 malaria parasites. Genome biology 9, R177

617 28. Jiang, C. and Pugh, B.F. (2009) A compiled and systematic reference map of 618 nucleosome positions across the Saccharomyces cerevisiae genome. Genome biology 10, 619 R109 
620 29. Tolstorukov, M.Y., et al. (2009) Comparative analysis of H2A.Z nucleosome

621 organization in the human and yeast genomes. Genome research 19, 967-977

622 30. Raisner, R.M., et al. (2005) Histone variant H2A.Z marks the 5' ends of both active 623 and inactive genes in euchromatin. Cell 123, 233-248

624 31. Guillemette, B., et al. (2005) Variant histone H2A.Z is globally localized to the

625 promoters of inactive yeast genes and regulates nucleosome positioning. PLoS biology 3,

626 e384

627 32. Karmodiya, K., et al. (2012) H3K9 and H3K14 acetylation co-occur at many gene 628 regulatory elements, while $\mathrm{H} 3 \mathrm{~K} 14 \mathrm{ac}$ marks a subset of inactive inducible promoters in 629 mouse embryonic stem cells. BMC genomics 13,424

630 33. Kouzarides, T. (2007) Chromatin modifications and their function. Cell 128, 693-705

631 34. Ragoczy, T., et al. (2006) The locus control region is required for association of the 632 murine beta-globin locus with engaged transcription factories during erythroid

633 maturation. Genes Dev 20, 1447-1457

634 35. Tolhuis, B., et al. (2002) Looping and interaction between hypersensitive sites in the 635 active beta-globin locus. Mol Cell 10, 1453-1465

636 36. Carter, D., et al. (2002) Long-range chromatin regulatory interactions in vivo. Nature 637 genetics 32, 623-626

638 37. Deng, W., et al. (2014) Reactivation of developmentally silenced globin genes by 639 forced chromatin looping. Cell 158, 849-860

640 38. Palstra, R.J., et al. (2003) The beta-globin nuclear compartment in development and 641 erythroid differentiation. Nature genetics 35, 190-194

642 39. Murrell, A., et al. (2004) Interaction between differentially methylated regions

643 partitions the imprinted genes Igf 2 and H19 into parent-specific chromatin loops. Nature 644 genetics $36,889-893$

645 40. Dean, A. (2011) In the loop: long range chromatin interactions and gene regulation.

646 Brief Funct Genomics 10, 3-10

647 41. de Wit, E. and de Laat, W. (2012) A decade of 3C technologies: insights into nuclear 648 organization. Genes Dev 26, 11-24

649 42. Dixon, J.R., et al. (2012) Topological domains in mammalian genomes identified by 650 analysis of chromatin interactions. Nature 485, 376-380

651 43. Nora, E.P., et al. (2013) Segmental folding of chromosomes: a basis for structural and 652 regulatory chromosomal neighborhoods? Bioessays $35,818-828$ 
653 44. Hou, C., et al. (2012) Gene density, transcription, and insulators contribute to the

654 partition of the Drosophila genome into physical domains. Mol Cell 48, 471-484

655 45. Li, L., et al. (2015) Widespread rearrangement of 3D chromatin organization 656 underlies polycomb-mediated stress-induced silencing. Mol Cell 58, 216-231

657 46. Bolzer, A., et al. (2005) Three-dimensional maps of all chromosomes in human male

658 fibroblast nuclei and prometaphase rosettes. PLoS biology 3, e157

659 47. Boyle, S., et al. (2001) The spatial organization of human chromosomes within the 660 nuclei of normal and emerin-mutant cells. Hum Mol Genet 10, 211-219

661 48. Cremer, T., et al. (2006) Chromosome territories--a functional nuclear landscape. 662 Curr Opin Cell Biol 18, 307-316

663 49. Croft, J.A., et al. (1999) Differences in the localization and morphology of

664 chromosomes in the human nucleus. J Cell Biol 145, 1119-1131

665 50. Federico, C., et al. (2006) Gene-rich and gene-poor chromosomal regions have 666 different locations in the interphase nuclei of cold-blooded vertebrates. Chromosoma 115, $667 \quad 123-128$

668 51. Foster, H.A. and Bridger, J.M. (2005) The genome and the nucleus: a marriage made 669 by evolution. Genome organisation and nuclear architecture. Chromosoma 114, 212-229

670 52. Zink, D., et al. (1998) Structure and dynamics of human interphase chromosome 671 territories in vivo. Hum Genet 102, 241-251

672 53. Lanctot, C., et al. (2007) Dynamic genome architecture in the nuclear space:

673 regulation of gene expression in three dimensions. Nat Rev Genet 8, 104-115

674 54. Bohmdorfer, G. and Wierzbicki, A.T. (2015) Control of Chromatin Structure by Long 675 Noncoding RNA. Trends in cell biology 25, 623-632

676 55. Maclary, E., et al. (2013) Long nonoding RNAs in the X-inactivation center.

677 Chromosome research : an international journal on the molecular, supramolecular and 678 evolutionary aspects of chromosome biology 21, 601-614

679 56. Callebaut, I., et al. (2005) Prediction of the general transcription factors associated 680 with RNA polymerase II in Plasmodium falciparum: conserved features and differences 681 relative to other eukaryotes. BMC genomics 6,100

682 57. Gangloff, Y.G., et al. (2001) The histone fold is a key structural motif of transcription 683 factor TFIID. Trends in biochemical sciences 26, 250-257

684 58. Flueck, C., et al. (2010) A major role for the Plasmodium falciparum ApiAP2 protein 685 PfSIP2 in chromosome end biology. PLoS pathogens 6, e1000784 
59. Ay, F., et al. (2014) Three-dimensional modeling of the P. falciparum genome during the erythrocytic cycle reveals a strong connection between genome architecture and gene expression. Genome research 24, 974-988

689 60. Freitas-Junior, L.H., et al. (2000) Frequent ectopic recombination of virulence factor 690 genes in telomeric chromosome clusters of P. falciparum. Nature 407, 1018-1022

691 61. Salcedo-Amaya, A.M., et al. (2009) Dynamic histone H3 epigenome marking during 692 the intraerythrocytic cycle of Plasmodium falciparum. Proceedings of the National 693 Academy of Sciences of the United States of America 106, 9655-9660

694 62. Crowley, V.M., et al. (2011) Heterochromatin formation in bistable chromatin 695 domains controls the epigenetic repression of clonally variant Plasmodium falciparum 696 genes linked to erythrocyte invasion. Molecular microbiology 80, 391-406

697 63. Freitas-Junior, L.H., et al. (2005) Telomeric heterochromatin propagation and histone 698 acetylation control mutually exclusive expression of antigenic variation genes in malaria 699 parasites. Cell 121, 25-36

700 64. Chookajorn, T., et al. (2007) Epigenetic memory at malaria virulence genes.

701 Proceedings of the National Academy of Sciences of the United States of America 104, $702 \quad 899-902$

703 65. Lopez-Rubio, J.J., et al. (2009) Genome-wide analysis of heterochromatin associates 704 clonally variant gene regulation with perinuclear repressive centers in malaria parasites.

705 Cell host \& microbe 5, 179-190

706 66. Miller, L.H., et al. (1994) Malaria pathogenesis. Science 264, 1878-1883

707 67. Scherf, A., et al. (2008) Antigenic variation in Plasmodium falciparum. Annual 708 review of microbiology $62,445-470$

709 68. Perez-Toledo, K., et al. (2009) Plasmodium falciparum heterochromatin protein 1 710 binds to tri-methylated histone 3 lysine 9 and is linked to mutually exclusive expression 711 of var genes. Nucleic acids research 37, 2596-2606

712 69. Flueck, C., et al. (2009) Plasmodium falciparum heterochromatin protein 1 marks

713 genomic loci linked to phenotypic variation of exported virulence factors. PLoS

714 pathogens 5 , e1000569

715 70. Duraisingh, M.T., et al. (2005) Heterochromatin silencing and locus repositioning

716 linked to regulation of virulence genes in Plasmodium falciparum. Cell 121, 13-24

717 71. Tonkin, C.J., et al. (2009) Sir2 paralogues cooperate to regulate virulence genes and 718 antigenic variation in Plasmodium falciparum. PLoS biology 7, e84

719 72. Coleman, B.I., et al. (2014) A Plasmodium falciparum histone deacetylase regulates 720 antigenic variation and gametocyte conversion. Cell host \& microbe 16, 177-186 
73. Jiang, L., et al. (2013) PfSETvs methylation of histone H3K36 represses virulence genes in Plasmodium falciparum. Nature 499, 223-227

723 74. Ukaegbu, U.E., et al. (2014) Recruitment of PfSET2 by RNA polymerase II to variant 724 antigen encoding loci contributes to antigenic variation in P. falciparum. PLoS pathogens $725 \quad 10, \mathrm{e} 1003854$

726 75. Brancucci, N.M., et al. (2014) Heterochromatin protein 1 secures survival and 727 transmission of malaria parasites. Cell host \& microbe 16, 165-176

728 76. Lopez-Rubio, J.J., et al. (2007) 5' flanking region of var genes nucleate histone 729 modification patterns linked to phenotypic inheritance of virulence traits in malaria 730 parasites. Molecular microbiology 66, 1296-1305

731 77. Volz, J.C., et al. (2012) PfSET10, a Plasmodium falciparum methyltransferase, 732 maintains the active var gene in a poised state during parasite division. Cell host \& 733 microbe $11,7-18$

734 78. Epp, C., et al. (2009) Chromatin associated sense and antisense noncoding RNAs are 735 transcribed from the var gene family of virulence genes of the malaria parasite

736 Plasmodium falciparum. Rna 15, 116-127

737 79. Amit-Avraham, I., et al. (2015) Antisense long noncoding RNAs regulate var gene 738 activation in the malaria parasite Plasmodium falciparum. Proceedings of the National

739 Academy of Sciences of the United States of America 112, E982-991

740 80. Broadbent, K.M., et al. (2011) A global transcriptional analysis of Plasmodium 741 falciparum malaria reveals a novel family of telomere-associated lncRNAs. Genome 742 biology 12, R56

743 81. Raabe, C.A., et al. (2010) A global view of the nonprotein-coding transcriptome in 744 Plasmodium falciparum. Nucleic acids research 38, 608-617

745 82. Sierra-Miranda, M., et al. (2012) Two long non-coding RNAs generated from 746 subtelomeric regions accumulate in a novel perinuclear compartment in Plasmodium 747 falciparum. Molecular and biochemical parasitology 185, 36-47

748 83. Luke, B. and Lingner, J. (2009) TERRA: telomeric repeat-containing RNA. The 749 EMBO journal 28, 2503-2510

750 84. Merrick, C.J., et al. (2012) Epigenetic dysregulation of virulence gene expression in 751 severe Plasmodium falciparum malaria. The Journal of infectious diseases 205, 1593-

$752 \quad 1600$

753 85. Abdi, A.I., et al. (2016) Global selection of Plasmodium falciparum virulence antigen 754 expression by host antibodies. Scientific reports 6, 19882 
755 86. Cowman, A.F. and Crabb, B.S. (2006) Invasion of red blood cells by malaria

756 parasites. Cell 124, 755-766

757 87. Cortes, A., et al. (2007) Epigenetic silencing of Plasmodium falciparum genes linked

758 to erythrocyte invasion. PLoS pathogens 3, e107

759 88. Josling, G.A., et al. (2015) A Plasmodium Falciparum Bromodomain Protein

760 Regulates Invasion Gene Expression. Cell host \& microbe 17, 741-751

761 89. Saraf, A., et al. (2016) Dynamic and Combinatorial Landscape of Histone

762 Modifications during the Intraerythrocytic Developmental Cycle of the Malaria Parasite.

763 Journal of proteome research 15, 2787-2801

764 90. Garcia, B.A., et al. (2007) Organismal differences in post-translational modifications

765 in histones H3 and H4. The Journal of biological chemistry 282, 7641-7655

766 91. Barski, A., et al. (2007) High-resolution profiling of histone methylations in the

767 human genome. Cell 129, 823-837

768 92. Bernstein, B.E., et al. (2005) Genomic maps and comparative analysis of histone

769 modifications in human and mouse. Cell 120, 169-181

770 93. Kim, T.H., et al. (2005) A high-resolution map of active promoters in the human

771 genome. Nature 436, 876-880

772 94. Nishida, H., et al. (2006) Histone H3 acetylated at lysine 9 in promoter is associated 773 with low nucleosome density in the vicinity of transcription start site in human cell.

774 Chromosome research : an international journal on the molecular, supramolecular and

775 evolutionary aspects of chromosome biology 14, 203-211

776 95. Wang, Z., et al. (2008) Combinatorial patterns of histone acetylations and

777 methylations in the human genome. Nature genetics 40, 897-903

778 96. Bartfai, R., et al. (2010) H2A.Z demarcates intergenic regions of the plasmodium

779 falciparum epigenome that are dynamically marked by H3K9ac and H3K4me3. PLoS

780 pathogens 6 , e 1001223

781 97. Cui, L., et al. (2007) PfGCN5-mediated histone H3 acetylation plays a key role in

782 gene expression in Plasmodium falciparum. Eukaryotic cell 6, 1219-1227

783 98. Trelle, M.B., et al. (2009) Global histone analysis by mass spectrometry reveals a 784 high content of acetylated lysine residues in the malaria parasite Plasmodium falciparum.

785 Journal of proteome research 8, 3439-3450

786 99. Ponts, N., et al. (2011) Nucleosome occupancy at transcription start sites in the 787 human malaria parasite: a hard-wired evolution of virulence? Infection, genetics and

788 evolution : journal of molecular epidemiology and evolutionary genetics in infectious

789 diseases $11,716-724$ 
100. Bunnik, E.M., et al. (2014) DNA-encoded nucleosome occupancy is associated with transcription levels in the human malaria parasite Plasmodium falciparum. BMC

792 genomics 15,347

793 101. Westenberger, S.J., et al. (2009) Genome-wide nucleosome mapping of Plasmodium

794 falciparum reveals histone-rich coding and histone-poor intergenic regions and chromatin

795 remodeling of core and subtelomeric genes. BMC genomics 10, 610

796 102. Ponts, N., et al. (2010) Nucleosome landscape and control of transcription in the

797 human malaria parasite. Genome research 20, 228-238

798 103. Kensche, P.R., et al. (2016) The nucleosome landscape of Plasmodium falciparum

799 reveals chromatin architecture and dynamics of regulatory sequences. Nucleic acids

800 research $44,2110-2124$

801 104. Schep, A.N., et al. (2015) Structured nucleosome fingerprints enable high-resolution 802 mapping of chromatin architecture within regulatory regions. Genome research 25, 1757-

$803 \quad 1770$

804 105. Lee, C.K., et al. (2004) Evidence for nucleosome depletion at active regulatory

805 regions genome-wide. Nature genetics 36, 900-905

806 106. Pokholok, D.K., et al. (2005) Genome-wide map of nucleosome acetylation and 807 methylation in yeast. Cell 122, 517-527

808 107. Mavrich, T.N., et al. (2008) Nucleosome organization in the Drosophila genome.

809 Nature 453, 358-362

810 108. Valouev, A., et al. (2008) A high-resolution, nucleosome position map of C. elegans

811 reveals a lack of universal sequence-dictated positioning. Genome research 18, 1051-

8121063

813 109. Beh, L.Y., et al. (2015) DNA-guided establishment of nucleosome patterns within 814 coding regions of a eukaryotic genome. Genome research 25, 1727-1738

815 110. Hoeijmakers, W.A., et al. (2013) H2A.Z/H2B.Z double-variant nucleosomes inhabit 816 the AT-rich promoter regions of the Plasmodium falciparum genome. Molecular

817 microbiology 87, 1061-1073

818 111. Petter, M., et al. (2013) H2A.Z and H2B.Z double-variant nucleosomes define 819 intergenic regions and dynamically occupy var gene promoters in the malaria parasite

820 Plasmodium falciparum. Molecular microbiology 87, 1167-1182

821 112. Le Roch, K.G., et al. (2004) Global analysis of transcript and protein levels across

822 the Plasmodium falciparum life cycle. Genome research 14, 2308-2318

823 113. Oehring, S.C., et al. (2012) Organellar proteomics reveals hundreds of novel nuclear 824 proteins in the malaria parasite Plasmodium falciparum. Genome biology 13, R108 
825 114. Lieberman-Aiden, E., et al. (2009) Comprehensive mapping of long-range

826 interactions reveals folding principles of the human genome. Science 326, 289-293

827 115. Ralph, S.A., et al. (2005) Antigenic variation in Plasmodium falciparum is 828 associated with movement of var loci between subnuclear locations. Proceedings of the

829 National Academy of Sciences of the United States of America 102, 5414-5419

830 116. Dahan-Pasternak, N., et al. (2013) PfSec13 is an unusual chromatin-associated 831 nucleoporin of Plasmodium falciparum that is essential for parasite proliferation in 832 human erythrocytes. Journal of cell science 126, 3055-3069

833 117. Dekker, J. (2016) Mapping the 3D genome: Aiming for consilience. Nature reviews. 834 Molecular cell biology 17, 741-742

835 118. Duan, Z., et al. (2010) A three-dimensional model of the yeast genome. Nature 465, $836 \quad 363-367$

837 119. Tanizawa, H., et al. (2010) Mapping of long-range associations throughout the 838 fission yeast genome reveals global genome organization linked to transcriptional 839 regulation. Nucleic acids research 38, 8164-8177

840 120. Weiner, A., et al. (2011) 3D nuclear architecture reveals coupled cell cycle 841 dynamics of chromatin and nuclear pores in the malaria parasite Plasmodium falciparum.

842 Cellular microbiology 13, 967-977

843 121. Chaal, B.K., et al. (2010) Histone deacetylases play a major role in the 844 transcriptional regulation of the Plasmodium falciparum life cycle. PLoS pathogens 6, 845 e1000737

846 122. Malmquist, N.A., et al. (2012) Small-molecule histone methyltransferase inhibitors 847 display rapid antimalarial activity against all blood stage forms in Plasmodium 848 falciparum. Proceedings of the National Academy of Sciences of the United States of 849 America 109, 16708-16713

850 123. Malmquist, N.A., et al. (2015) Histone methyltransferase inhibitors are orally 851 bioavailable, fast-acting molecules with activity against different species causing malaria 852 in humans. Antimicrobial agents and chemotherapy 59, 950-959

853 124. Dekker, J., et al. (2002) Capturing chromosome conformation. Science 295, 1306$854 \quad 1311$

855 125. Gondor, A., et al. (2008) High-resolution circular chromosome conformation 856 capture assay. Nature protocols 3, 303-313

857 126. Dostie, J., et al. (2006) Chromosome Conformation Capture Carbon Copy (5C): a 858 massively parallel solution for mapping interactions between genomic elements. Genome 859 research $16,1299-1309$

860 


\section{Outstanding Questions}

1. Does the nucleus of $P$. falciparum harbor one or more repressive heterochromatin clusters at the nuclear periphery?

2. Are the epigenetic control mechanisms observed throughout the asexual life cycle similar in other stages?

3. Through what mechanisms do stage-specific transcription factors control the transition between specific developmental stages?

4. Which chromatin-associated proteins are vital for maintaining the epigenetics features and the 3D structure of the parasite nucleus and can these key proteins be targeted for development of antimalarial drug therapies?

5. What is the role of epigenetic control in gene regulation in other Plasmodium species? 


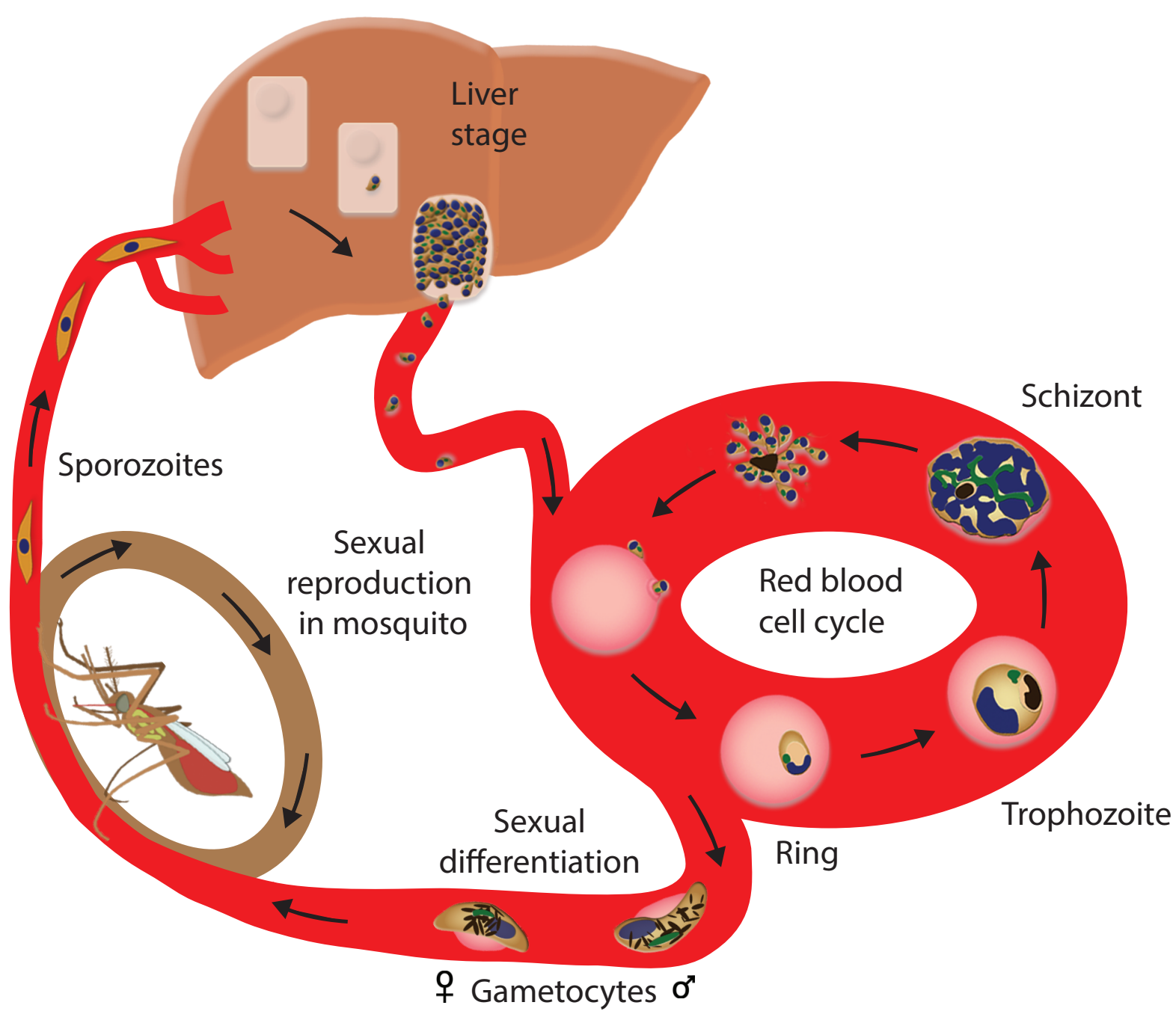




\section{Figure 2:}

A Vargenes

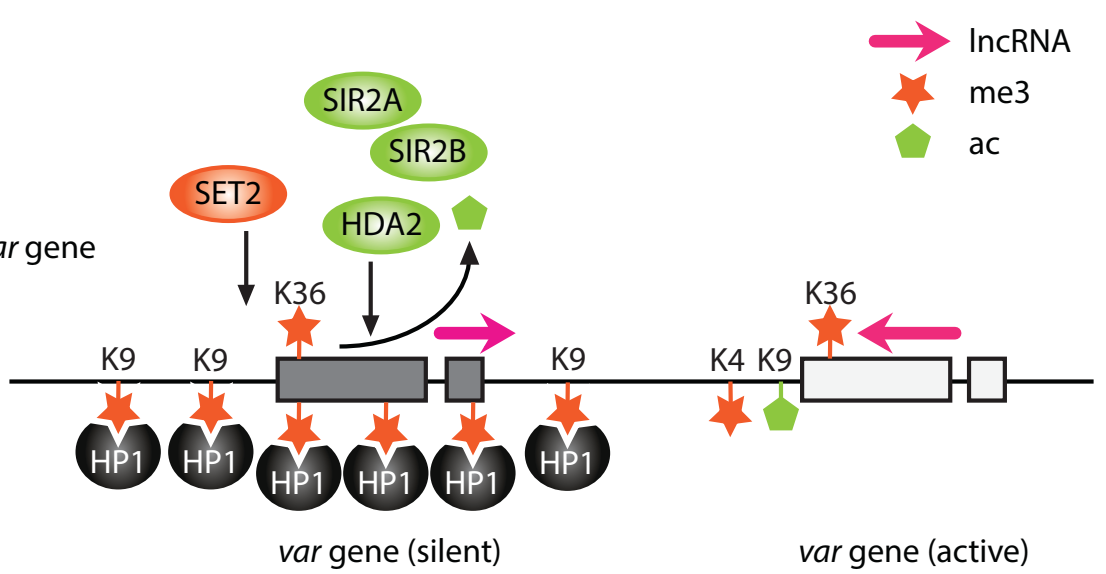

B Invasion genes

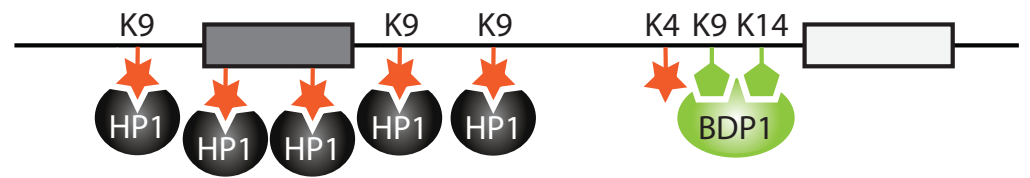

Invasion gene (silent)

Invasion gene (active)

C Gametocyte transcription factor

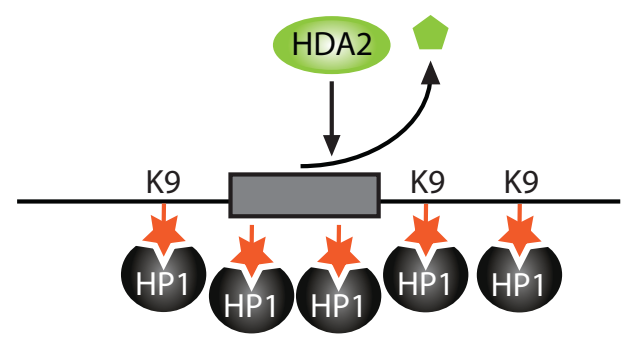

pfap2-g (silent)



pfap2-g (active) 
Figure 3:

\section{Saccharomyces cerevisiae}

A

AT content

$2 \%$

B

Nucleosome landscape



C

Nucleosome levels during transcription

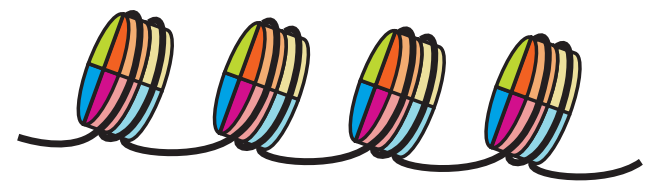

D

Chromosome conformation

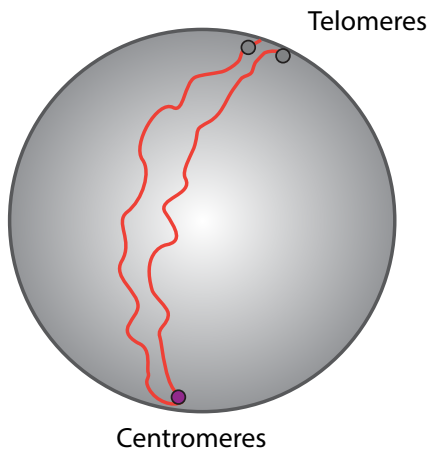

Plasmodium falciparum

GC

$19 \%$

$81 \%$
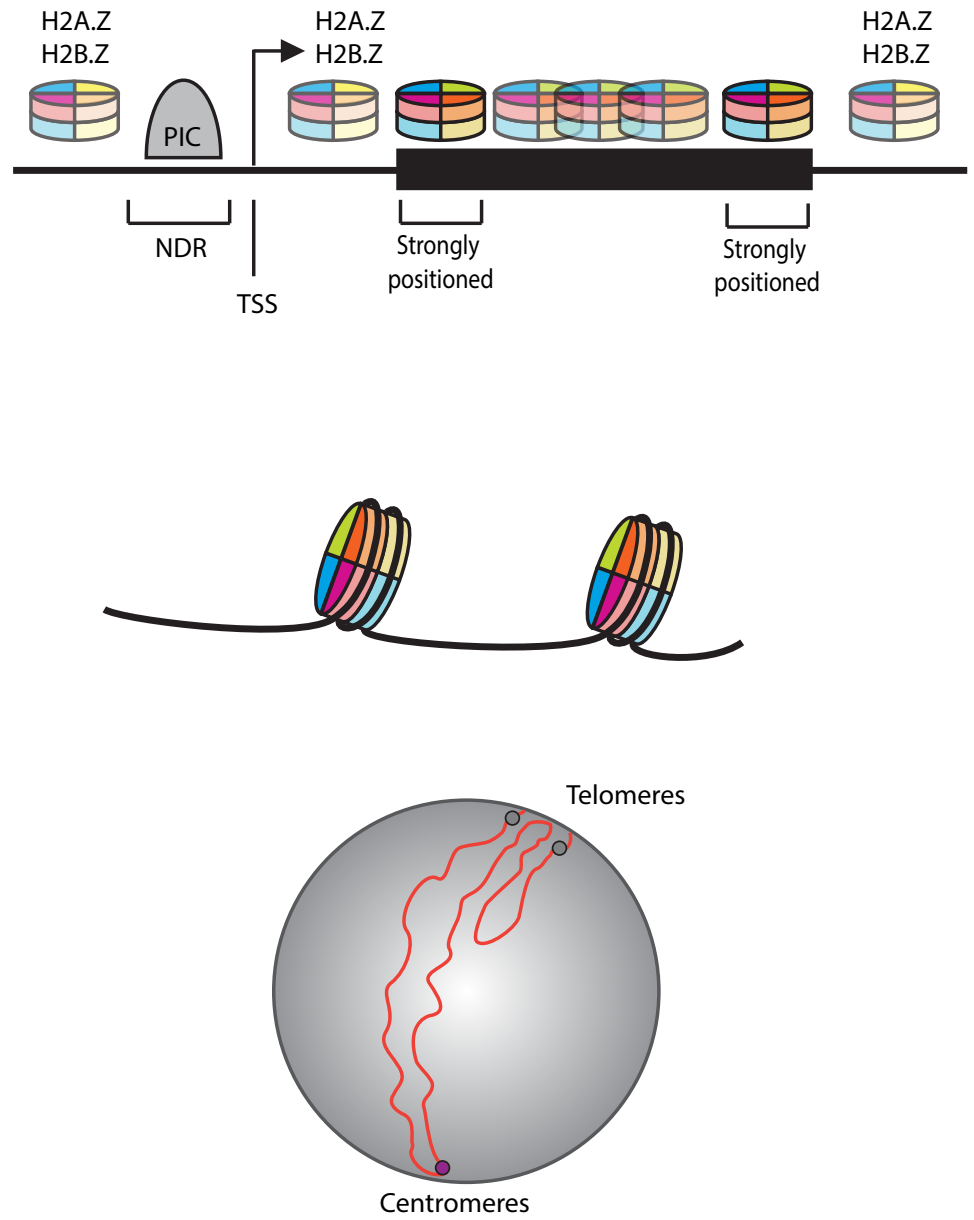